\title{
Gusto y disposición por la innovación científico-tecnológica en estudiantes mexicanos de educación media superior
}

\author{
Javier Tarango, Irma Guajardo-Morales, Juan D. Machin-Mastromatteo* y Armando Villanueva-Ledezma \\ Facultad de Filosofía y Letras, Universidad Autónoma de Chihuahua, México. Rúa de las Humanidades s/n Ciudad \\ Universitaria, 31174 Chihuahua, Chih., México (e-mail: jtarango@uach.mx, irmguajardo@hotmail.com, \\ jmachin@uach.mx, avillanueva@uach.mx).
}

* Autor a quien debe ser dirigida la correspondencia

Recibido May. 24, 2019; Aceptado Jul. 22, 2019; Versión final Ago. 15, 2019, Publicado Feb. 2020

\begin{abstract}
Resumen
Este estudio analiza la percepción de 4456 estudiantes de bachillerato del estado de Chihuahua (México), en relación al gusto (entendido como el interés personal) y disposición (el deseo de continuar participando) hacia la innovación científico-tecnológica. El objetivo del estudio es determinar la factibilidad de generar ambientes de formación académica extracurricular (clubes de ciencias). Mediante una encuesta, se recolectaron datos que permitieron identificar en los sujetos de estudio los siguientes momentos: 1) experiencias previas de aplicación científica y de autoconcepto sobre creatividad y personas creativas; 2) intereses actuales en la innovación científico-tecnológica; 3) conocimientos y habilidades; y 4) disposición de participación activa en ambientes científicos. Los resultados muestran que, al ser analizados los cuatro momentos como un método, sólo poblaciones menores indican constancia en gusto y disposición a involucrarse en eventos académicos relacionados con la innovación.
\end{abstract}

\section{Preference and willingness for scientific-technological innovation in Mexican high school students}

\begin{abstract}
This study analyzes the perception of 4456 high school students from the state of Chihuahua (Mexico), in relation to their preferences (understood as personal interest) and willingness (the desire to continue participating) towards scientific-technological innovation. The objective is determining the feasibility of generating extracurricular academic training environments (science clubs). The information was collected using a questionnaire and the data allowed the identification of the following moments in the study subjects: 1) previous experiences of scientific application and self-concept about creativity and creative people; 2 ) current interests in scientific-technological innovation; 3) knowledge and skills; and 4) provision of active participation in scientific environments. The results show that, when the four moments are analyzed as a method, only minor populations indicate constancy in taste and willingness to get involved in academic events related to innovation, which justifies the feasibility of opening promotional spaces for the science; On the other hand, a large population offers results of disinterest and lack of willingness to participate.
\end{abstract}

Keywords: scientific education; scientific training; technological innovation; intellectual curiosity 


\section{INTRODUCCIÓN}

Esta investigación partió de la premisa de que la sociedad actual está sufriendo una transformación académica especialmente en la estructura de los planes de estudio, atribuida principalmente a los avances tecnológicos y científicos que se experimentan, con un preocupante ritmo acelerado que se incorpora en la dinámica de los sistemas educativos. Tal situación se caracteriza por la inclusión de procesos y funciones relacionados con las tecnologías de la información (como el correo electrónico, videoconferencias, chat o los metabuscadores) al proceso de enseñanza y aprendizaje (Ummah et al., 2019), con fines de facilitar la construcción del aprendizaje en forma práctica y carente de una reflexión, basado todo ello en la emergencia de los procesos de creatividad para identificar situaciones reales y retos sociales (Pifarré, 2019), lo cual en ocasiones no sucede, debido a diversas circunstancias de acceso, uso y apropiación de tales medios.

Los acelerados cambios provocados por la tecnología suelen repetirse en todos los ámbitos de la educación, tal es el caso de la ciencia y la innovación tecnológica, donde se hacen esfuerzos por posicionarlas en el gusto de los adolescentes, entendido este concepto como la demostración del interés personal y motivación. Sin embargo, los resultados no necesariamente han sido gratificantes, ya que cuando se realizan esfuerzos para incrementar los niveles de cultura científica en estudiantes (investigación científica, creatividad, innovación y cambios en formas de pensamiento científico), no se logra percibir un incremento sustancial. Tales condiciones sucedan, se deberá incluir al estudiante desde niveles básicos, en dinámicas académicas vinculadas en comunidades de práctica, cuya línea de trabajo relacione a la investigación con la enseñanza y gusto por la ciencia y la tecnología (Lucchiari et al., 2019; Srikoon et al., 2018).

Debe considerarse que los propósitos de las instituciones educativas por incrementar los niveles de creatividad e innovación no necesariamente muestran concordancia con los objetivos académicos de los propios estudiantes (Revelo et al., 2017). Por ejemplo, la resistencia de los jóvenes al enfrentar las materias académicas vinculadas a las ciencias exactas o las ciencias experimentales, regularmente se manifiesta en dificultades para entenderlas y acreditarlas. Incluso, este tipo de condiciones se atribuyen a factores familiares relacionados con el entorno que a su vez contribuyen en la creatividad, tales como: estatus socioeconómico, tamaño y estructura de la familia (Jankowskaa y Karwowskib, 2019); estos son factores sobre los cuales los sistemas educativos no tienen influencia o control, especialmente los públicos.

La creatividad e innovación no debe manejarse como una condición asociativa con los conocimientos técnicocientíficos vinculados a las ciencias exactas, sino que debe mostrar una relación real con todas las disciplinas del saber humano. Por ejemplo, las ciencias sociales y las humanidades implican muchos elementos relacionados con la creatividad y la innovación, sin necesariamente tener relación con el uso de equipos tecnológicos específicos (Morales, 2017). Las tendencias educativas actuales proponen explorar la interacción entre la ciencia, artes y la creatividad inherente para tener un mejor entendimiento en el potencial de la enseñanza y el aprendizaje, por tanto, la propuesta común es combinar ciencia-tecnología-ingenieríamatemáticas o más allá, volver transdisciplinario a la ciencia-tecnología-ingeniería-artes-matemáticas (Chappell et al., 2019).

El desarrollo de la cultura científica ofrece una amplia gama de contribuciones en el campo educativo, abriendo un abanico de posibilidades en cuanto a modalidades formativas, que suelen situarse dentro del ámbito de la creatividad e innovación, sobre todo en la posibilidad de establecer relaciones de trabajo colaborativo. Esto permite usar formas de enseñanza más acordes con las demandas de los nuevos modelos educativos. La innovación y la creatividad ofrecen nuevas perspectivas hacia el estudio de la ciencia, incluyendo procesos de reflexión y de comunicación interpersonal (Bustamante, 2014). Consecuencia de esto, es que las instituciones educativas deben experimentar los mismos cambios al exigirse desarrollar habilidades creativas e innovativas, mismas que puedan influir, tanto en ambientes futuros de los estudiantes de EMS: ocupacionales y en la educación superior (Vally et al., 2019).

El objetivo de este trabajo es evaluar y clasificar los rasgos de cultura científica de estudiantes de EMS en relación con sus gustos y condiciones de disposición por la innovación científico-tecnológica, con lo cual sea posible justificar el desarrollo de ambientes de formación académica extracurricular. Para lograr este objetivo, es necesario identificar distintas condiciones y características sobre: 1) Experiencias y conocimientos previos; 2) Intereses personales; 3) Concepciones y autoconcepciones sobre conocimiento propio, habilidades personales en relación con la creatividad y la innovación; y 5) Disposiciones personales en relación con el involucramiento futuro en ambientes que propicien el desarrollo de ciencia y tecnología.

\section{CREATIVIDAD E INNOVACIÓN EN LA CIENCIA Y TECNOLOGÍA}

La incorporación de los estudiantes ambientes relacionados con las tecnologías puede realizarse a través de tres enfoques fundamentales de formación diferenciada: 1) obtención de las nociones básicas de la tecnología; 2) profundización en conocimientos incrementando capacidades para resolver problemas reales pensados de forma sistémica; y 3) creación de conocimiento a través de la innovación (UNESCO, 2016). Por 
tanto, resulta fundamental considerar que los estudiantes y docentes deberán generar procesos de cultura científica, ya que su desarrollo, especialmente en la EMS, propicia que el estudiante adquiera conocimientos, aplicando los principios de la ciencia, tecnología, ingeniería y matemáticas en situaciones prácticas de su vida cotidiana. La promoción en el desarrollo de habilidades y conocimientos interdisciplinarios se convierten en un medio de contribución al gusto por la ciencia, tecnología, innovación, economía y competitividad internacionales (Hsu-Chan et al., 2019), contribuyendo al desarrollo como ciudadanos globales, caracterizados por sus niveles de conciencia, cuidado y aceptación de la diversidad cultural, al tiempo que se promueve la justicia social y la sostenibilidad, junto con un sentido de responsabilidad para actuar (Tidikis y Dunbar, 2019).

Los estudiantes deben convivir en entornos que propicien la cultura científica, entendida como el conjunto total de los actos humanos en una comunidad en relación con las actividades científicas (incluye la comprensión de la ciencia, la investigación científica, los ambientes de productividad científica, así como la producción y comunicación de la ciencia). Esto involucra prácticas científicas y tecnológicas que buscan la formación integral del ser humano, dando prioridad al logro del desarrollo económico, social, cultural y académico de las personas y los países. Este concepto comprende otros, como la comunicación científica, difusión del conocimiento o 'public understanding' que en ocasiones se ha convertido, según Lévy Leblond (2003), en la 'guerra de la ciencia' manifestándose en tres formas: 1) el modelo lineal de la ciencia a través de un proceso acumulativo del conocimiento; 2) la descalificación profesional en relación con la investigación científica; y 3) la pérdida de la pertinencia de los temas estudiados y su relevancia a corto y mediano plazo. Ante todo, debe considerarse que los estudiantes particularmente de generaciones jóvenes, deben estar preparados para enfrentar los retos de la 4a revolución industrial, la cual implica lo siguiente: las habilidades de pensamiento creativo, la solución de problemas y el desarrollo de una amplia capacidad adaptativa ante los cambios (Hidayat et al., 2018), situaciones demandadas en los mercados laborales actuales a través de procesos de implementación de la innovación abierta, donde se propicia la colaboración y la inteligencia colectiva (Álvarez-Aros y Bernal-Torres, 2017).

La promoción de la ciencia en estudiantes y profesores debe iniciarse con el conocimiento y manejo del método científico en aplicaciones básicas, en temas relacionados con el ambiente y en las ciencias naturales, buscando que sus entornos de aprendizaje incluyan laboratorios. Sin embargo, los resultados esperados a largo plazo deberían tender hacia el desarrollo de la capacidad de autoaprendizaje, el aprendizaje permanente, la estructura de procesos de cognición y metacognición, así como a la interpretación y aplicación de conocimientos científicos den su vida cotidiana en referencia a casos reales y condiciones sociales (Peñaherrera et al., 2013; Ulger, 2019; Villamizar et al., 2013).

Las acciones de promoción de la ciencia y la tecnología permiten identificar patrones actitudinales de vocación científica y tecnológica, al grado que las actividades científicas extraclase resulten de mayor interés, a través de predicciones positivas, tales como: 'la ciencia me gusta más que otras asignaturas', 'la ciencia ha aumentado mi curiosidad', 'la ciencia ha aumentado mi aprecio por la naturaleza', 'podemos encontrar soluciones a los problemas científicos', por mencionar las principales. Para Merino (2008), el pensamiento creativo de los adolescentes, en relación con su orientación vocacional, está determinado por la necesidad de: generar alternativas, romper estereotipos personales, ambientales y ocupacionales, la exploración creativa de las alternativas, tomar decisiones adecuadas y generar conciencia sobre limitaciones personales, sin olvidar que existen elementos en contra como los bloqueos culturales y emocionales que inhiben el desarrollo del individuo.

Impulsar la ciencia y tecnología en estudiantes jóvenes ofrece múltiples ventajas, pero presenta infinidad de implicaciones, las cuales ponen en riesgo la posibilidad de implantar modelos de formación académica y al mismo tiempo, dificultan los resultados reales esperados. Algunas implicaciones importantes son las siguientes: 1) medir la creatividad en contextos escolares es difícil, especialmente cuando los instrumentos disponibles no ofrecen la suficiente confiabilidad y validez; 2) el gusto por la ciencia está determinado por su relación con las variables de autoconcepto y creatividad, ya que el autoconcepto favorece a la formación íntegra de las personas, permitiendo el desarrollo y potencial creativo de todos; 3) la creatividad es un tema de estudio que puede llegar a ser controversial, ya que está vinculado con aspectos personales, sociales, culturales e incluso, económicos (Franco, 2016; Ruano, 2017).

La creatividad tiene que ver con la ejecución de acciones de las personas en contextos muy diversos y con su funcionamiento óptimo, la innovación, la solución de problemas de todo tipo, los avances científicos y tecnológicos, y los cambios sociales. En definitiva, la creatividad puede ser considerada como una de las características más importantes del ser humano y por tanto de sus producciones; 4) existe una fuerte influencia de las TIC en la innovación de los estudiantes y en la práctica docente de los profesores, siendo un medio para resolver problemas, además, se considera a una persona con amplia educación aquella que tiene una vasta relación con las tecnologías actuales, mismas que son los medios más eficientes de transmisión de datos y las personas eficientes no son concebidas como aquellas que crean, sino que utilizan lo ya creado (Marcelo, 2013); y 5) Los procesos de creatividad en relación con la ciencia, la tecnología y la innovación no 
surgen por generación espontánea, sino que están basados en un conocimiento profundo sobre la problemática a resolver, permitiendo reconstruirla en sus partes o elementos para obtener una mirada diferente y novedosa que ofrezca soluciones, a partir de la motivación personal (Krumm et al., 2013; Moote, 2019).

Existen tres tipos de creatividad, dependiendo de si se trata de generar procesos o productos inherentes a un interés personal. Esta clasificación comprende los siguientes tipos (Pineda et al., 2017): 1) creatividad objetiva y realista, vinculada con el ambiente del estudiante; 2) creatividad imaginaria y fantástica, la cual surge más allá de la realidad propia del sujeto, no tiene un modelo lógico y se basa en la imaginación; y 3) creatividad innovadora e inventiva, relacionada con el cambio y mejora de la realidad. Tristán y Mendoza (2016) presentan otra clasificación de la creatividad, basados en los siguientes estilos: 1) transformador (actividades hacia la mejora); 2) visionario (búsqueda de soluciones ideales a largo plazo); 3) experimental (cambio de ideas que se pueden probar); y 4) explorador (uso de las percepciones para encontrar solución a problemas.

La creatividad en la ciencia ofrece elementos de complejidad, demanda disciplina y fundamentos cognitivos para solucionar los problemas, no se puede caer en el romanticismo, ya que muchos estudiantes provienen del desarrollo sistemático de procesos educativos vinculados a la inhibición al aprendizaje de las ciencias (Stolte et al., 2019). Los jóvenes de EMS poseen su propio imaginario sobre la ciencia y la tecnología, entre los que se destacan): 1) imaginario colectivo como conocimientos altamente complejos; 2) representación acerca de los científicos y las carreras de esta profesión como personas fuera de un contexto moderno, incluso conceptualizadas como personas disfuncionales; 3) valoración del aporte de las materias científicas en distintos aspectos de la vida, considerándolas altamente útiles para el desarrollo social y el mejoramiento de la calidad de vida, especialmente en la curación de las enfermedades; 4) posibilidad de formar hábitos informativos sobre ciencia y tecnología; y 5) caracterización socio-cultural de la ciencia, relacionado con un estatus medio en la escala de actividades de éxito en la vida (Krumm et al., 2013). Las percepciones hacia la ciencia, tecnología e innovación de estudiantes del nivel medio y medio superior demandan promover una actitud científica por parte de las instituciones educativas, esto significa, buscar que adopten formas de acercamiento a problemas, la indagación y la experimentación científica (Calderón, 2015).

La existencia de procesos de formación en cultura científica, particularmente en la EMS, radica en que el estudiante debe alcanzar un conocimiento general del universo y sus elementos en el sentido más amplio, lo cual a su vez es básico para la cultura del hombre moderno (Arteta, 2009). Por tanto, los sistemas educativos demandan acciones específicas para la enseñanza de la ciencia y tecnología, no tanto por cuestiones de su ejecución, sino para asegurar que se aporte al desarrollo de una visión de cultura general. El estudio de la ciencia comprende las acciones pedagógicas desarrolladas, de forma específica en el salón de clase, pero sobrepasa tales límites, llegando de manera fundamental a permearse en todos los ámbitos institucionales. Los sistemas educativos actuales luchan para que la cultura científica permee en los ámbitos social, regional y del propio sector educativo, en todos los niveles que lo componen (Li y Geng, 2016). Esto demanda implantar nuevos programas académicos, reformar los ya existentes o buscar alternativas extracurriculares para que suceda, a través de acciones basadas en la motivación del estudiante que se proyecten en la acción (Sunley et al., 2019).

La EMS se considera el último nivel académico del adolescente dentro de un proceso educativo escolarizado, previo a afrontar la educación superior. En este nivel, se propone la formación integral del ser humano, por medio de vivencias que potencialicen el pensamiento creativo. La generación de elementos que influyen el gusto por la creatividad y la innovación se convierte en un aporte a esa formación integral. Esta clase de propuestas se alinean a la propia teoría educativa, a las condiciones actuales de la educación básica en cualquier contexto regional y la definición de los propios propósitos de la sociedad de la información y el conocimiento; situaciones que se han convertido en el estandarte para la definición del sujeto ideal para afrontar las demandas sociales actuales y futuras.

\section{METODOLOGÍA}

Las variables de interés para esta investigación son: 1) Variable dependiente: nivel de estudiantes de EMS en relación con la innovación científico-tecnológica; y 2) Variables independientes: gusto y disposición por la innovación científico-tecnológica y factibilidad de creación de ambientes para propiciar la creatividad y la innovación.

Tipo de estudio. Se orientó a un diseño de investigación de enfoque cuantitativo y descriptivo, donde se relacionaron las condiciones existentes, opiniones o actividades a partir del estudio de una muestra de estudiantes de EMS. Por su tipo, esta investigación se caracterizó por ser: 1) exploratoria, por lo que fue un estudio preliminar y de una cohorte específica; 2) realizada para recoger mayor información respecto a un problema; 3) un mecanismo que sirve principalmente, para aclarar conceptos y conocer dimensiones centrales de un problema; y 4) una investigación inicial y preparatoria, ya que se realizó para recoger datos y precisar su naturaleza. 
Muestreo. Por tratarse de un diseño transversal no experimental, la muestra no probabilística se basó en la totalidad de los estudiantes de EMS que se encontraban cursando el tercer semestre de bachillerato de los planteles seleccionados (ciclo escolar agosto-diciembre, 2017). La muestra estudiada consistió en 4,456 estudiantes de EMS, quienes durante la recolección de datos se encontraban cursando el tercer semestre de bachillerato.

Espacio. Se eligió para desarrollar el estudio al Colegio de Bachilleres del Estado de Chihuahua, México, bajo las siguientes consideraciones: 1 ) es un sistema educativo compuesto de distintos planteles que abarca las principales zonas urbanas del Estado de Chihuahua, México, atendiendo a masas de población estudiantil en el nivel medio superior en la categoría de bachillerato general bivalente (47,000 estudiantes, aproximadamente); 2) ser una institución pública, con buen nivel de aceptación y preferencia por parte de estudiantes y padres de familia, como la primera opción para cursar la EMS; y 3) ofrecer un plan de estudios donde, al cursar el tercer semestre, el estudiante tiene la posibilidad de elegir materias propedéuticas relacionadas con las ciencias sociales o las ciencias exactas.

Instrumento de recolección de datos. Se utilizó la técnica de la encuesta, misma que se aplicó de forma que, el estudiante dentro de sus clases, accediera a una computadora y ofreciera sus respuestas, las cuales se concentraron posteriormente en SPSS. La encuesta estuvo compuesta por los siguientes apartados: 1) datos de control (nombre, plantel, semestre, turno y sexo); 2) condiciones previas (se evaluó en dos preguntas: la aplicación previa de elementos de la ciencia y la tecnología; y el autoconcepto de la persona creativa); 3) identificación de intereses (se evaluó en dos preguntas: el gusto por el diseño; reparación y por armar y desarmar); 4) identificación de conocimientos y habilidades (se evaluó en una pregunta: conocimiento de programación); y 5) disposición hacia la participación (se evaluó en cuatro preguntas: el interés por la integración de equipos de ciencia y tecnología, así como el desarrollo de habilidades). En general, las preguntas fueron cerradas, con dos opciones de respuesta (si/no) y abiertas.

Modelo conceptual simplificado. A partir de los aspectos que se evaluaron y de las condiciones del instrumento de recolección de datos, se elaboró un modelo conceptual simplificado (véase Figura 1), donde se integran los elementos y determinan la forma de análisis de los datos.

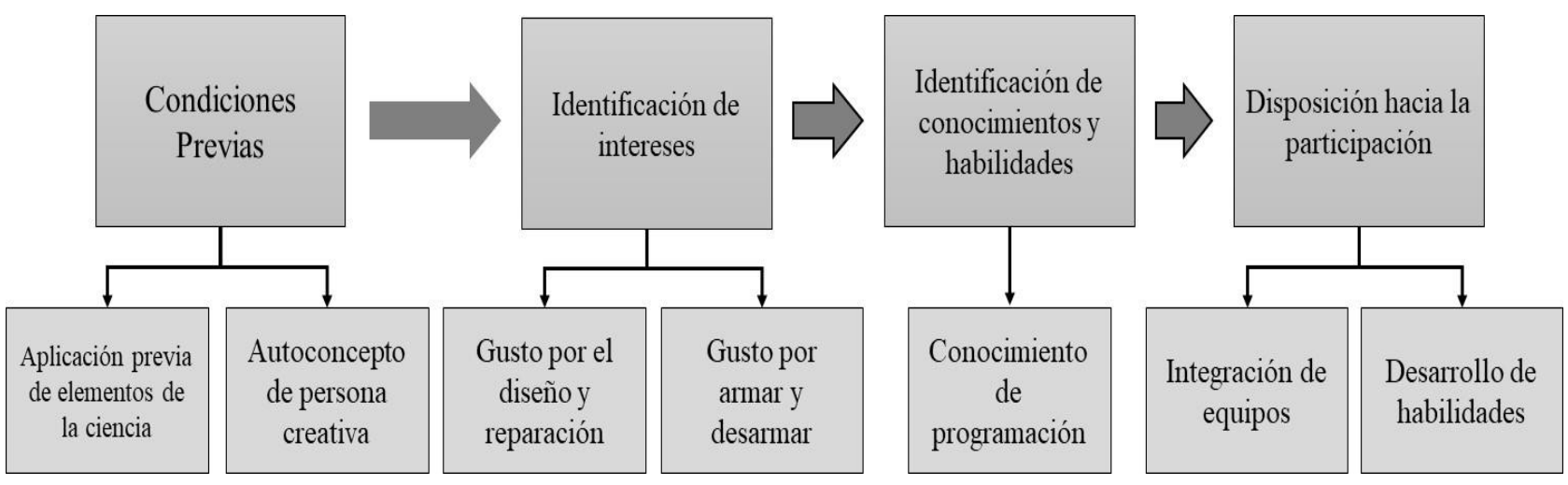

Fig. 1: Modelo conceptual simplificado

\section{RESULTADOS Y DISCUSIÓN}

Se encuestó la población total de los grupos existentes en los planteles elegidos, los cuales fueron seis planteles, tres en la ciudad de Chihuahua, México y tres en Ciudad Juárez, México, obteniéndose los siguientes resultados:

\section{Condiciones actuales}

Este apartado en general se refiere a una serie de cuestionamientos hechos a los estudiantes con el fin de indagar su participación previa o su estado actual en la generación de algún invento, primero, concretándose en el afán de conocer la parte proporcional de la población estudiada en cuanto a en qué medida han tenido participación actual o previa en actividades científicas y tecnológicas; y posteriormente, se identificaron los tipos de inventos en los que se ha participado (véase Tabla 1).

Según los resultados obtenidos, ante la pregunta si han inventado algo aplicando elementos de la ciencia y tecnología (como por ejemplo carritos o robots), según los valores expuestos, se observa que sólo el $17.1 \%$ tiene o ha tenido una experiencia científica y el $83.1 \%$ no manifiesta experiencia al respecto. Los inventos en los que han estado involucrados los estudiantes participantes en el estudio fueron 25 alternativas, lo cual demuestra una gama amplia de opciones provenientes de poblaciones limitadas. 
Tabla 1: Tipo de invento en que ha estado involucrado el estudiante

\begin{tabular}{|c|c|c|}
\hline Tipo de invento & Frecuencia & Porcentaje \\
\hline Autos, aviones, helicópteros u otros juguetes sin movimiento & 194 & 25.70 \\
\hline Autos, barcos u otros juguetes con energía solar, eléctrica o eólica a control remoto & 112 & 14.83 \\
\hline Robots y brazos de robots & 99 & 13.11 \\
\hline Catapulta & 94 & 12.45 \\
\hline Productos como jabón, porta teclados, bebidas, instrumentos musicales o cajas musicales & 77 & 10.20 \\
\hline Aparatos eléctricos o electrónicos & 32 & 4.24 \\
\hline Lámparas (solares, con paneles, eléctricas) & 30 & 3.97 \\
\hline Software, computadoras, calculadoras, celulares, USB, laser y controles & 20 & 2.65 \\
\hline Elementos de decoración, collares, pulseras, portarretratos, cepillos o ropa & 18 & 2.38 \\
\hline Prototipos, maquetas y modelos a escala & 13 & 1.72 \\
\hline Autos, autopartes y motores & 13 & 1.72 \\
\hline Amplificadores de audio, de sonido, o estéreos con luces rítmicas & 12 & 1.59 \\
\hline Cajas de descargas eléctricas & 7 & 0.93 \\
\hline Aparatos mecánicos & 7 & 0.93 \\
\hline Legos & 5 & 0.66 \\
\hline Videojuegos & 5 & 0.66 \\
\hline Generación de electricidad a través de aspas & 4 & 0.53 \\
\hline Bicicletas & 4 & 0.53 \\
\hline Mecanismos de salud & 3 & 0.40 \\
\hline Bici bomba (tanque de agua) & 1 & 0.13 \\
\hline Carro limpia ductos & 1 & 0.13 \\
\hline Locomotoras y ciudades en miniatura & 1 & 0.13 \\
\hline Máquina para hacer quesos y otros productos & 1 & 0.13 \\
\hline Máquinas de combustión interna & 1 & 0.13 \\
\hline Sensores & 1 & 0.13 \\
\hline Total & 755 & 100.00 \\
\hline
\end{tabular}

\section{Identificación de intereses}

Aquí se identifican las intenciones de los estudiantes por participar en actividades relacionadas con la ciencia y la tecnología, lo cual se divide en diversos aspectos, tales como: deseos de contribuir en la creación de ciertos aparatos móviles; clasificación de los tipos de artefactos en los que se desea participar; identificación del gusto por armar o desarmar aparatos; y los tipos de aparatos que les gustarían armar o desarmar. En relación con el gusto por construir, diseñar o reparar artefactos de partes móviles, se observó que sólo el $29.9 \%$ de la población estudiantil participante tienen gusto por hacerlo y al $70.1 \%$ de ellos no les gusta. En relación con los tipos de artefactos de partes móviles en los que le gustaría participar en la construcción, diseño o rediseño, se preguntó a los participantes que lo expresaran de forma libre y se identificaron 21 tipos, cuya variedad denota amplitud de opciones identificadas por los participantes (véase Tabla 2).

Tabla 2: Tipo de artefacto de partes móviles que les gustaría construir, diseñar o rediseñar

\begin{tabular}{|l|c|c|}
\hline \multicolumn{1}{|c|}{ Tipo de artefacto } & Frecuencia & Porcentaje \\
\hline Aparatos eléctricos o electrónicos & 365 & 28.23 \\
\hline Software, computadoras, calculadoras, celulares, USB, laser y controles & 294 & 22.74 \\
\hline Carritos, aviones, helicópteros u otros juguetes sin movimiento & 134 & 10.36 \\
\hline Autos, autopartes y motores & 134 & 10.36 \\
\hline Robots y brazos de robots & 122 & 9.44 \\
\hline Carros, barcos u otros juguetes con energía solar, eléctrica o eólica a control remoto & 48 & 3.71 \\
\hline Aparatos mecánicos & 41 & 3.17 \\
\hline Amplificadores de audio, de sonido o estéreos con luces rítmicas & 37 & 2.86 \\
\hline Prototipos, maquetas y modelos a escala & 28 & 2.17 \\
\hline Productos como porta teclados, instrumentos musicales o cajas musicales & 22 & 1.70 \\
\hline Máquinas de combustión interna & 16 & 1.24 \\
\hline Videojuegos & 12 & 0.93 \\
\hline Legos & 8 & 0.62 \\
\hline
\end{tabular}


Tabla 2 (Continuación)

\begin{tabular}{|l|c|c|}
\hline \multicolumn{1}{|c|}{ Tipo de artefacto } & Frecuencia & Porcentaje \\
\hline Elementos de decoración, collares, pulseras, portarretratos, cepillos o ropa & 7 & 0.54 \\
\hline Bicicletas & 6 & 0.46 \\
\hline Relojes & 5 & 0.39 \\
\hline Generación de electricidad a través de aspas & 4 & 0.31 \\
\hline Lámparas (solares, con paneles o eléctricas) & 4 & 0.31 \\
\hline Cajas de descargas eléctricos & 3 & 0.23 \\
\hline Mecanismos de salud & 2 & 0.15 \\
\hline Locomotoras y ciudades en miniatura & 1 & 0.08 \\
\hline Total & 1,293 & 100.00 \\
\hline
\end{tabular}

Se cuestionó además a los participantes en cuanto a su gusto por armar y desarmar aparatos de todo tipo, para este cuestionamiento las respuestas recolectadas fueron más favorables, ya que, en este criterio, el $44.4 \%$ indicó mostrar interés, en tanto que el $55.6 \%$ indicó lo contrario. Nuevamente, a través de una pregunta abierta, se solicitó de los participantes indicar libremente el tipo de soporte que le gustaría armar o desarmar (véase Tabla 3), donde se identificaron 20 tipos, considerándose dos renglones de alta relevancia: software, computadoras, calculadoras, celulares, USB, laser y controles, y aparatos eléctricos o electrónicos, siendo el resto opciones de baja frecuencia.

Tabla 3: Tipo de aparatos que les gustaría armar o desarmar

\begin{tabular}{|l|c|c|}
\hline \multicolumn{1}{|c|}{ Tipo de aparatos } & Frecuencia & Porcentaje \\
\hline Software, computadoras, calculadoras, celulares, USB, laser y controles & 792 & 40.53 \\
\hline Aparatos eléctricos o electrónicos & 670 & 34.29 \\
\hline Videojuegos & 85 & 4.35 \\
\hline Autos, autopartes y motores & 80 & 4.09 \\
\hline Carritos, aviones, helicópteros u otros juguetes sin movimiento & 64 & 3.28 \\
\hline Amplificadores de audio, de sonido o estéreos con luces rítmicas & 46 & 2.35 \\
\hline Carros, barcos u otros juguetes con energía solar, eléctrica o eólica con control remoto & 46 & 2.35 \\
\hline Aparatos mecánicos & 44 & 2.25 \\
\hline Robots y brazos de robots & 41 & 2.10 \\
\hline Productos como porta teclados, instrumentos musicales o cajas musicales & 1.59 \\
\hline Legos & 31 & 0.77 \\
\hline Máquinas de combustión interna & 15 & 0.51 \\
\hline Relojes & 10 & 0.46 \\
\hline Elementos de decoración, collares, pulseras, portarretratos, cepillos o ropa & 9 & 0.26 \\
\hline Lámparas (solares, con paneles o eléctricas) & 5 & 0.26 \\
\hline Prototipos, maquetas y modelos a escala & 5 & 0.20 \\
\hline Bicicletas & 4 & 0.15 \\
\hline Rompecabezas & 3 & 0.10 \\
\hline Cajas de descargas eléctricos & 2 & 0.05 \\
\hline Locomotoras y ciudades en miniatura & 2 \\
\hline Total & 1 & 0.05 \\
\hline
\end{tabular}

Tanto en las Tablas 2 y 3 , es posible identificar múltiples opciones en las que el estudiante tendría intenciones de participar, sin embargo, es posible que en cada caso las frecuencias se concentren en pocas alternativas, correspondiendo a cinco y tres opciones respectivamente hasta completar el $80 \%$ del total de los gustos manifiestos, el resto puede considerarse irrelevantes a partir de su baja frecuencia.

\section{Identificación de conocimientos y habilidades}

En este rasgo de evaluación, se estudian percepciones sobre conocimientos y habilidades tecnológicas que el sujeto participante considera poseer. Siguiendo el mismo modelo de análisis de los criterios previamente evaluados, para este caso se partió de identificar si se poseen conocimientos de programación computacional o de videojuegos; posteriormente, se cuestionó a los estudiantes sobre su autoconcepto sobre si posee creatividad; y se le solicitó que comentara su concepción propia de creatividad en una pregunta abierta. Los resultados recolectados indican que los estudiantes participantes que tienen conocimientos de programación computacional o en videojuegos fue el $21 \%$, mientras que el $79 \%$ indicó no tener conocimientos al respecto. La autoconcepción de los estudiantes respecto a si se consideran personas creativas, se observó que el $70.3 \%$ sí se consideró creativo y el $29.7 \%$ no creyó tener tal cualidad. 
Ante la pregunta sobre qué entienden los participantes por creatividad, se les solicitó expresar su propio concepto de forma libre, con lo cual se identificaron coincidencias las cuales fueron conjuntadas en cinco propuestas (véase Tabla 4): 1) un enfoque hacia innovación, evitando guiarse por lo que ya está creado, pero sobre todo ofreciendo soluciones concretas, correspondiendo esta respuesta al $44.95 \%$ del total; 2) ninguna conceptualización, correspondiente al $30.03 \%$ del total (estas dos primeras opciones suman el $74.98 \%$ del total); 3) enfocado a elementos inusuales, pero que no necesariamente ofrezcan soluciones; 4) tendencias innovadoras más aplicadas al diseño, puede decirse que comprende un enfoque artístico o estético, incluso comprende no sólo aparatos sino comprende también la literatura por ejemplo; y 5) desarrollo de adecuaciones a los elementos ya creados con fines a la mejora.

Tabla 4: Definiciones de creatividad

\begin{tabular}{|c|c|c|c|}
\hline Definición de creatividad & Frecuencia & Porcentaje & $\begin{array}{l}\text { Porcentaje } \\
\text { acumulado }\end{array}$ \\
\hline $\begin{array}{l}\text { 1. Creación de cosas nuevas sin copiar de las que ya existen y } \\
\text { que solucionen un problema concreto }\end{array}$ & 2,003 & 44,95 & 44.95 \\
\hline 2. Ninguna conceptualización & 1,338 & 30,03 & 74.98 \\
\hline 3. Imaginación, innovación y creación de cosas inusuales & 843 & 18,92 & 93,90 \\
\hline $\begin{array}{l}\text { 4. Aplicación de la estética en la creación, diseño o dibujo, } \\
\text { definiendo tendencias }\end{array}$ & 210 & 4,71 & 98.61 \\
\hline $\begin{array}{l}\text { 5. Desarrollar adecuaciones o diferencias a partir de las cosas ya } \\
\text { creadas, que perfeccionen el producto }\end{array}$ & 62 & 1,39 & 100,00 \\
\hline Total & 4456 & 100,00 & \\
\hline
\end{tabular}

De acuerdo a los resultados anteriores llama en especial la atención que un porcentaje elevado de estudiantes son incapaces de expresar alguna conceptualización propia sobre la creatividad, aunque previamente respondieron reconocer ser personas creativas.

\section{Identificación de la disposición}

Ante la propuesta de investigar diversos elementos de un proceso de diagnóstico sobre las condiciones de la ciencia y la tecnología en los entornos de estudiantes de EMS, luego de haber estudiado la experiencia previa, el conocimiento y el gusto por este tema, se procedió a analizar la disposición de los sujetos en cuanto a su participación extracurricular en actividades científicas y tecnológicas. La primera pregunta planteada, referente a la identificación de disposición, se refirió a si se tiene el gusto por ser parte de un equipo para la fabricación de aparatos, mecanismos o máquinas completas, de lo cual, el $38.8 \%$ respondió de forma positiva y el $61.2 \%$ manifestó de forma negativa. Al cuestionar a los estudiantes participantes en el estudio si estarían interesados en formar parte del equipo tecno-científico para la fabricación de retos tecnológicos, el 39.4\% respondió de forma positiva y el $60.6 \%$ en forma negativa.

Otro cuestionamiento planteado respecto a la identificación de la disposición fue en relación al gusto por desarrollar habilidades y creatividad en la fabricación de prototipos mecánicos y robóticos, donde se mostró un incremento en los resultados positivos, alcanzándose un $45.6 \%$ para quienes manifestaron tener gusto por desarrollar esta clase de procesos de aprendizaje, mientras que el $54.4 \%$ dijo no estar interesado.

Respecto al total de los estudiantes encuestados y su interés en formar parte del club de ciencias e innovación tecnológica, el $39.1 \%$ están interesados en el tema y el $60.9 \%$ no. Posteriormente y sólo en relación a los estudiantes que, ante la pregunta anterior respondieron de forma positiva, se les cuestionó sobre su propuesta de horario para participar en eventos de ciencia y tecnología (véase Tabla 4), identificándose seis alternativas, siendo la más viable cuando se oferte en contra turno de los horarios de clase, pero no los sábados (54.18\%), seguida de la propuesta de horarios en sábados (31.29\%).

Tabla 5: Propuestas de horarios para participar en un club de ciencia e innovación tecnológica

\begin{tabular}{|l|c|c|c|}
\hline \multicolumn{1}{|c|}{ Propuesta de horario } & Frecuencia & Porcentaje & $\begin{array}{c}\text { Porcentaje } \\
\text { acumulado }\end{array}$ \\
\hline Contra turno, pero no los sábados & 1,541 & 54.18 & 54.18 \\
\hline Sábados & 890 & 31.29 & 85.47 \\
\hline Viernes & 188 & 6.61 & 92.08 \\
\hline Al terminar la jornada de clases & 108 & 3.80 & 95.88 \\
\hline Domingos & 101 & 3.55 & 99.43 \\
\hline Lunes a jueves & 16 & 0.56 & 100.00 \\
\hline Total & 2,844 & 100,00 & \\
\hline
\end{tabular}




\section{Correlación de variables}

Con el fin de ofrecer diversas comparaciones entre las dimensiones evaluados (propuestos en el modelo conceptual simplificado), se incluye una serie de correlaciones para demostrar si los estudiantes participantes en el estudio mantienen un comportamiento estable entre ellas, dando como resultado la identificación de condiciones de factibilidad en el establecimiento de escenarios para la ciencia, la tecnología y la innovación a partir de poblaciones suficientes para que sucedan. Para ofrecer datos más claros, sólo se presentan promedios de correlación de los criterios evaluados en cada dimensión (véase Tabla 6).

Tabla 6: Correlaciones entre variables evaluadas

\begin{tabular}{|l|l|c|}
\hline \multicolumn{1}{|c|}{ Dimensión previa } & \multicolumn{1}{|c|}{ Dimensión posterior } & $\begin{array}{c}\text { Nivel de } \\
\text { correlación }\end{array}$ \\
\hline Condiciones previas & $\begin{array}{l}\text { Identificación de } \\
\text { intereses }\end{array}$ & $.409^{\star *}$ \\
\hline $\begin{array}{l}\text { Identificación de } \\
\text { intereses }\end{array}$ & $\begin{array}{l}\text { Identificación de } \\
\text { conocimientos y } \\
\text { habilidades }\end{array}$ & $.195^{\star *}$ \\
\hline $\begin{array}{l}\text { Identificación de } \\
\text { conocimientos y } \\
\text { habilidades }\end{array}$ & $\begin{array}{l}\text { Disposición hacia la } \\
\text { participación }\end{array}$ & $.283^{\star *}$ \\
\hline
\end{tabular}

De acuerdo a los resultados de correlación, se observa que existe significancia entre las dimensiones evaluadas, lo que permite deducir que los estudiantes con experiencias previas conservan el interés en la ciencia y la tecnología, así como, aquellos que identifican sus conocimientos y habilidades, conservan disposición hacia el involucramiento futuro. Aunque los resultados son positivos, debe considerarse que sólo corresponden a porcentajes bajos de la población estudiada que respondieron a las preguntas de forma positiva, que en ninguno de los casos alcanzó el 50\% del total de la población estudiada.

\section{DISCUSIÓN}

La manifestación de la cultura científica en adolescentes puede suceder por cuestiones relacionadas con la propia personalidad y vocación del sujeto, situación que se proyecta a través del interés en la ciencia, por tanto, en el caso de los resultados en los que un alto porcentaje de estudiantes encuestados manifiestan no tener interés en este tipo de iniciativas, poco se puede hacer. Tal consideración está sustentada en la propuesta de Moote (2019), quien se basa en una perspectiva cognitiva social en donde el individuo está condicionado en actitudes autorreguladas hacia el aprendizaje y defiende su progreso académico, objetivos y estrategias. Esta perspectiva se caracteriza porque las instituciones educativas no tienen manera de influir en los estudiantes, ya que está determinada por factores familiares y sociales (Jankowskaa y Karwowskib, 2019).

Ante la búsqueda de estrategias para desarrollar la cultura científica, un aspecto en el que las instituciones educativas tienen fuerte influencia sobre los estudiantes es a través de los procesos educativos de educación formal, en donde el currículo académico define materias, contenidos y perfiles de formación. La promoción de la creatividad a través de acciones curriculares interdisciplinarias, diseño de actividades de interacción con la tecnología y cursos desarrollados de forma expresa facilitan la adquisición de la cultura científica (Hsu-Chan et al., 2019), especialmente cuando se combinan la ciencia, la tecnología y el arte (Chappell et al., 2019; Ulger, 2019). De acuerdo con las condiciones de la EMS mexicana, la incidencia directa en la formación en cultura científica a través del currículo formal resulta demasiado complejo de llevarse a la práctica, salvo a través de las intenciones positivas de profesores que lo hagan voluntariamente, sin alterar las condiciones temáticas de los planes de estudio.

Otra manera de fomentar la cultura científica, es el desarrollo de acciones provenientes de escenarios ad hoc, que de manera informal promuevan elementos para que suceda, a través de acciones de mejora de la creatividad en proyectos basados en aprendizaje (Ummah et al., 2019), observan el carácter de no obligatoriedad y en donde el participante muestra interés auténtico, sin embargo, ofrece la limitante de volverse excluyente, la cual no sólo puede referirse a personas con distintas discapacidades físicas o mentales, sino además, características personales de inhibición (Stolte et al., 2019), siendo que en proyectos de innovación siempre se fomenta la participación colectiva, sin respetar el individualismo por encima del colectivismo (Pifarré, 2019). La alternativa de acciones extracurriculares que involucren a los estudiantes de EMS en actividades científicas, puede resultar la opción más viable, ya que, aunque las poblaciones que muestran gusto y disposición por la innovación científico-tecnológica representen porcentajes inferiores, son las que mayor seguridad ofrecen para el establecimiento de escenarios de promoción de la ciencia.

El planteamiento inicial es esta investigación parte del interés de la institución bajo estudio, al pretender justificar la creación de clubes de ciencias en la EMS a través de manifestaciones de interés y disposición hacia la innovación científico-tecnológica, planteándose que sólo a través de la iniciativa de las propias 
organizaciones educativas pueden suceder esta clase de eventos (Lucchiari et al., 2019), sin embargo, prevalece el paradigma de enfocar esas iniciativas únicamente a las ciencias exactas y aplicadas, con lo cual se identifica una demanda manifiesta de considerar a la creatividad y a la innovación desde dos perspectivas fundamentales: 1) incluir la visión aplicada a las humanidades y las ciencias sociales; y 2) considerar diversos niveles de creatividad e innovación, que no sólo se relacionan con la generación de ideas nuevas, sino además, en relación con propuestas de mejora. De acuerdo a las concepciones de creatividad vertidas por los estudiantes que participaron en el estudio, estas son más amplias y existiendo una manifestación clara sobre la no exclusión, principalmente de las artes y humanidades.

La promoción de la interdisciplinariedad científica muestra una relación directa con actividades académicas que promueven la crítica por encima de la ejecución (Chappell et al., 2019), al cuestionarse que sólo así se puede contribuir al desarrollo del verdadero ciudadano que la sociedad global está demandando (Tidikis y Dunbar, 2019), considerando tres aspectos fundamentales en sus niveles de involucramiento: nivel de originalidad en la generación de múltiples alternativas y variadas soluciones; capacidad de elaboración de productos y fluidez en la generación de ideas o asociaciones para cualquier problema que se le presente ( $\mathrm{Li}$ y Geng, 2016; Vally et al., 2019), usando un pensamiento crítico y creativo y habilidades de solución de problemas, especialmente en cambios vertiginosos del conocimiento (Hidayat et al., 2018; Ulger, 2019). La innovación científico-tecnológica demanda de practicidad, pero además de capacidad crítica, sin esto último suele desanimar la participación de estudiantes con perfiles vinculados a las humanidades; aun en los ambientes de las ciencias exactas, el estudiante debe desarrollar su capacidad de persuasión, planeación, desempeño, producción y presentación de los resultados, demostrando así el uso correcto de los procesos de investigación, incorporando elementos de cooperación, libertad de ideas y aspectos éticos (Srikoon et al., 2018).

\section{CONCLUSIONES}

Una vez que se han evaluado y clasificado los rasgos de cultura científica en estudiantes de EMS, los gustos y condiciones de disposición por la innovación científico tecnológica ponen de manifiesto que, según los resultados, se justifica la decisión institucional de implantar espacios para promover la ciencia y la tecnología, especialmente cuando se identifican correlaciones estadísticas positivas entre las diferentes variables evaluadas. Sin embargo, es necesario ponderar condiciones específicas en cuanto a: disposición de infraestructura humana y tecnológica; acondicionamiento de espacios físicos de acuerdo a los tipos de aparatos o equipos que se pretenden desarrollar; y capacidad de promoción de los servicios que se ofrecerán, especialmente en poblaciones no interesadas en ellos.

Las acciones que se desarrollen con adolescentes para promover la cultura científica deben hacer explícito que la creatividad e innovación tecnológica no sólo corresponde a las ciencias exactas, sino que es parte de todas las profesiones y situaciones futuras de la vida. Se considera que, en la dinámica social actual, existe una tendencia fuerte al desarrollo económico relacionado con actividades laborales que demandan la aplicación de las ciencias tecnológicas, de profesionales vinculados a la innovación y creatividad y de formación de sujetos que contribuyan a las condiciones favorables para el desarrollo económico de la región y en particular de las personas.

\section{REFERENCIAS}

Álvarez-Aros, E.L., C.A. Bernal-Torres, Modelo de Innovación Abierta: Énfasis en el Potencial Humano, Información Tecnológica, doi: 10.4067/S0718-07642017000100007, 28(1), 65-76 (2017)

Arteta, J.R., Cultura Científica en el Sistema Educativo del Departamento de la Guajira Colombiana, Zona Próxima: Revista del Instituto de Estudios en Educación Universidad del Norte, ISSN: 1657-2416, 11, 102-121 (2009)

Bustamante, Y., La Educación Media Superior en México, Innovación Educativa, ISSN: 1665-2673, 14(64), 11-22 (2014)

Calderón, R., La Percepción de la Ciencia, Tecnología e Innovación en Estudiantes del Nivel Medio y Medio Superior de la Zona Metropolitana de Guadalajara, México, Revista Iberoamericana para la Investigación y el Desarrollo Educativo, ISSN: 2007-7467, 6(11), 1-21 (2015)

Chappell, K., L. Hetherington, H. Horin, K. Nikolopoulos, S. Sotiriou y F.X. Bogner, Dialogue and materiality/embodiment in science/arts creative pedagogy: Their role and manifestation, Thinking Skills and Creativity, https://doi.org/10.1016/j.tsc.2018.12.008, 31, 296-322 (2019)

Franco, C., Relación entre las Variables Autoconcepto y Creatividad en una Muestra de Alumnos de Educación Infantil, REDIE. Revista Electrónica de Investigación Educativa, ISSN: 1607-4041, 8(1), 1-16 (2016)

Hsu-Chan, K., T. Yuan-Chi y C.A. Ya-Ting, Promoting college student's learning motivation and creativity through a STEM interdisciplinary PBL human-computer interaction system design and development course, Thinking Skills and Creativity, https://doi.org/10.1016/j.tsc.2018.09.001, 31, 1-10 (2019)

Hidayat, T., E. Susilaningsih y C. Kurniawan, the effectiveness of enrichment test instruments design to measure students' creative thinking skills and problem-solving, Thinking Skills and Creativity, https://doi.org/10.1016/j.tsc.2018.02.011, 29, 161-169 (2018) 
Jankowskaa, D.M. y M. Karwowskib, Family Factors and Development of Creative Thinking. Personality and Individual Differences, https://doi.org/10.1016/j.paid.2018.07.030,142, 202-206 (2019)

Krumm, G. L., V. Arán y otros tres autores, Invariancia de Medidas de la Prueba de Figuras del Test de Pensamiento Creativo de Torrance según la Edad: Un Estudio en Niños y Adolescentes de Habla Hispana, Cuadernos de Neuropsicología / Panamerican Journal of Neuropsychology, ISSN: 0718-4123, 7(2), 29-49 (2013)

Lévy, J.M., Una Cultura sin Cultura: Reflexiones Críticas sobre la Cultura Científica, Revista CTS: Revista Iberoamericana de Ciencia, Tecnología y Sociedad, ISSN: 1668-0030, 1(1), 139-151 (2013)

Li, Z. y Q.X. Geng, The Application Research of Science and Technology Activities Month in the Cultivation of College Students' Innovative Practical Ability, Proceedings 7th International Conference on Information Technology in Medicine and Education (2016)

Lucchiari, C., P.M. Sala y M.E. Vanutelli, the effects of a cognitive pathway to promote class creative thinking. An experimental study on Italian primary school students, Thinking Skills and Creativity, https://doi.org/10.1016/j.tsc.2018.12.002, 31, 156-166 (2019)

Marcelo, C., Las Tecnologías para la Innovación y la Práctica Docente, Brasileira de Educação, ISSN: 1413-2478O, 18(52), 25-47 (2013)

Merino, E., El Pensamiento Creativo en la Orientación Vocacional de los Adolescentes, International Journal of Developmental and Educational Psychology, ISSN: 0214-9877, 4(1), 241-248 (2008)

Morales-Valiente, C., La Creatividad: Una Revisión Científica, Arquitectura y Urbanismo, ISSN 1815-5898, XXXVIII (2), 53-62 (2017)

Moote, J., Investigating the Longer-Term Impact of the CREST Inquiry-Based Learning Programme on Student SelfRegulated Processes and Related Motivations: Views of Students and Teachers, Res Sci Educ, DOI 10.1007/s11165-0179621-7, 201(4), 265-294 (2019)

Peñaherrera, M., A. Ortiz-Colón y F. Cobos-Alvarado, ¿Cómo Promover la Educación Científica en el Alumnado de Primaria? Una Experiencia desde el Contexto Ecuatoriano, Revista Eureka sobre Enseñanza y Divulgación de las Ciencias, ISSN-e: 1697-011X, 10(2), 222-232 (2013)

Pifarré, M., Using interactive technologies to promote a dialogic space for creating collaboratively: A study in secondary education, Thinking Skills and Creativity, https://doi.org/10.1016/j.tsc.2019.01.004, 32, 1-16 (2019)

Pineda, L.M., L. Uribe y E. Díaz, ¿Cómo se Entiende Formar para la Investigación en Contextos de Educación Media y de Educación Superior? Revista Científica Guillermo de Ockham, ISSN: 1794-192X, 5(2), 11-22 (2017)

Revelo, R.E., J.P. Pazmiño-Linzán, A.L. Mata-Velasteguí y S.A. Avilés-Mena, Estudio de los Beneficios de Tener una Estación Científica en la Amazonía en las Universidades. Caso: Universidad Central del Ecuador, Revista Publicando, ISSN-e: 1390-9304,10(1), 505-519 (2017)

Ruano, L.E., Límites y Ventajas en el Proceso de Enseñanza y Aprendizaje de la Investigación Formativa, Revista San Gregorio, ISSN-e: 1390-7247,16, Edición Especial, 143-153 (2017)

Srikoon, S., T. Bunterm, T. Nethanomsak y K.N. Tang, Effect of 5P Model on Academic Achievement, Creative Thinking, and Research Characteristics, https://doi.org/10.1016/j.kjss.2018.06.011, Kasetsart Journal of Social Science, 39(3), 488495 (2018)

Stolte, M., E.H. Kroesbergena y J.E.H. Van Luit, Inhibition, friend or foe? Cognitive inhibition as a moderator between mathematical ability and mathematical creativity in primary school students, Personality and Individual Differences, https://doi.org/10.1016/j.paid.2018.08.024, 142, 196-201 (2019)

Sunley, R., L. Harding y J. Jones, Realising Creativity in Management Education: Putting Student Energy into Action, The International Journal of Management Education, DOI: 10.1016/j.ijme.2019.02.007, 17(2), 172-181 (2019)

Tidikis, V. y N.D. Dunbar, Openness to experience and creativity: When does Global Citizenship Matter? International Journal of Psychology, DOI: 10.1002/ijop.12463. 54(2), 264-268 (2019)

Tristán, A. y L. Mendoza, Taxonomías sobre Creatividad, Revista de Psicología, ISSN-e: 1579-3680, 34(1), 147-183 (2016)

Ulger, K., Comparing the effects of art education and science education on creative thinking in high school students, Arts Education Policy Review, DOI: 10.1080/10632913.2017.1334612, 120(2), 57-79 (2019)

Ummah, S. K., In'am, A., y Azmi, R. D. Creating manipulatives: improving students' creativity through project-based learning, Journal on Mathematics Education, ISSN 2087-8885, 10(1), 93-102 (2019)

UNESCO, Competencias y Estándares TIC desde la Dimensión Pedagógica: Una Perspectiva desde los Niveles de Apropiación de las TIC en la Práctica Educativa Docente, 8-14. Universidad Javeriana; UNESCO, Cali, Colombia (2016)

Villamizar, R.M., R. Flores-Buils y M. García-Renedo, La Identidad Juvenil en Contextos de Conflicto: Una Doble Mirada por sí Mismos y por los Otros, Fórum de RECERCA, http://dx.doi.org/10.6035/ForumRecerca.2013.33, 18, 491-504 (2013)

Vally, Z., L. Sallaoum y otros cinco autores, Examining the effects of creativity training on creative production, creative selfefficacy, and neuro-executive functioning, Thinking Skills and Creativity, https://doi.org/10.1016/j.tsc.2018.11.003, 30, 7078 (2019) 
